Mycobacterium tuberculosis-infected individuals: associations with clinical disease state and effect of treatment. J Immunol 2001; 167: 5217-5225.

3 Carrara S, Vincenti D, Petrosillo N, et al. Use of a T cell-based assay for monitoring efficacy of antituberculosis therapy. Clin Infect Dis 2004; 38: 754-756.

4 Kobashi Y, Mouri K, Fukuda M, et al. Transitional change in the clinical features of pulmonary tuberculosis. Respiration 2008; 75: 304-309.

5 Bosshard V, Roux-Lombard P, Perneger T, et al. Do results of the T-SPOT.TB interferon- $\gamma$ release assay change after treatment of tuberculosis? Respir Med 2009; 103: 30-34.

6 Mack U, Migliori GB, Sester M, et al. LTBI: latent tuberculosis infection or lasting immune responses to $M$. tuberculosis? A TBNET consensus statement. Eur Respir J 2009; 33: 956-973.
7 Wu-Hsieh BA, Chen CK, Chang JH, et al. Long-lived immune response to early secretory antigenic target 6 in individuals who had recovered from tuberculosis. Clin Infect Dis 2001; 33: 1336-1340.

8 Ribeiro S, Dooley K, Hackman J, et al. T-SPOT.TB responses during treatment of pulmonary tuberculosis. BMC Infect Dis 2009; 9: 23.

9 Dheda K, Pooran A, Pai M, et al. Interpretation of Mycobacterium tuberculosis antigen-specific IFN- $\gamma$ release assays (T-SPOT.TB) and factors that may modulate test results. J Infect 2007; 55: 169-173.

10 Elias D, Britton S, Kassu A, et al. Chronic helminth infections may negatively influence immunity against tuberculosis and other diseases of public health importance. Expert Rev Anti Infect Ther 2007; 5: 475-484.

\title{
Tracheal granuloma pyogenicum with erlotinib treatment for lung cancer
}

\section{To the Editors:}

We present the case of a 64 yr-old ex-smoker (20 pack-yrs) with a squamous cell carcinoma of the lung who developed a tracheitis and granuloma pyogenicum during treatment with erlotinib. Among the specific side-effects of epidermal growth factor receptor (EGFR) inhibitors, paronychia with granuloma pyogenicum has been reported frequently; however, granuloma pyogenicum has never been described in the trachea with erlotinib treatment.

Monoclonal antibodies against the EGFR (e.g. cetuximab) and EGFR tyrosine receptor inhibitors (TKIs) (e.g. gefinitib and erlotinib) have been shown to improve treatment outcomes in nonsmall cell lung cancer. Generally, these agents are better tolerated than classical cytotoxic agents showing a different toxicity profile. Specific side-effects of EGFR-targeting agents include diarrhoea and particularly skin toxicity, such as acneiform eruptions, xerosis, eczema, fissures, teleangiectasia, hyperpigmentation, hair changes and paronychia with granuloma pyogenicum [1]. Skin toxicity has been associated with EGFR inhibitor antitumor activity [1].

A 64 yr-old male was diagnosed with squamous cell carcinoma of the right upper lobe and was treated with a right upper lobectomy in December 2002 (pathological (p)T1 pN1 (three positive lymph nodes out of 33 removed)). No adjuvant treatment was given at that time. In 2007, the patient was treated with a right lower lobe resection due to recurrence; the histology confirmed a squamous cell carcinoma (retreatment classification (r)pT1b rpN0 R0). In 2009, he developed a second recurrence in the right mediastinal lymph nodes (stations $4 \mathrm{R}$ and 7) and was treated with a rest-pneumonectomy, showing three out of three positive lymph nodes with angiosis carcinomatosa (rpT1a rpN2 R0). The patient received four cycles of adjuvant cisplatin/vinorelbine, but developed progression in the mediastinal lymph nodes immediately after chemotherapy. Thus, palliative treatment with erlotinib $(150 \mathrm{mg})$ was initiated in
October 2009. He developed acneiform skin lesions of moderate intensity, as typically seen during therapy with a TKI, and achieved disease stabilisation with erlotinib for almost a year. In June 2010, he developed a vigorous dry cough that was refractory to various treatments, including codeine, steroids (systemic and inhaled) and $\mathrm{H}_{2}$ blockers.

In order to further investigate the cause of the cough, a bronchoscopy was performed, which showed a severe acute and chronic tracheobronchitis with significant breath-dependent tracheobronchial collapse and multiple white granular mucosal lesions of the distal trachea, which were biopsied (fig. 1).

To our surprise, the histopathological work-up showed no evidence of tumour, which was initially suspected, but small nodular lesions with granulation tissue and fibrinous material at the surface (which are white in colour when viewed on endoscopy), consistent with granuloma pyogenicum (fig. 2).

As no other explanation for the tracheal granuloma pyogenicum and the agonising cough was identified, treatment with erlotinib was stopped in July 2010, resulting in improvement of the cough within 2 weeks and complete remission of the granulomas in the follow-up bronchoscopy 6 weeks later (fig. 3).

5 weeks later, due to the progression of the mediastinal lymph nodes, a tracheal stent was placed; during the course of the disease, mediastinal radiotherapy with 30 Gy was performed due to further lymph node progression. The patient is currently undergoing further chemotherapy due to systemic progression but has had no recurrence of cough attacks.

Granuloma pyogenicum (also known as eruptive haemangioma, granulation tissue-type haemangioma, granuloma gravidarum and lobular capillary haemangioma [2]) is a polypoid form of capillary haemangioma. It is hypothesised to be a nonspecific tissue reaction seen after various insults but pathogenesis is controversial (neoplasm versus reactive hyperplasia) [3]. It occurs on either the skin or at the mucosal 


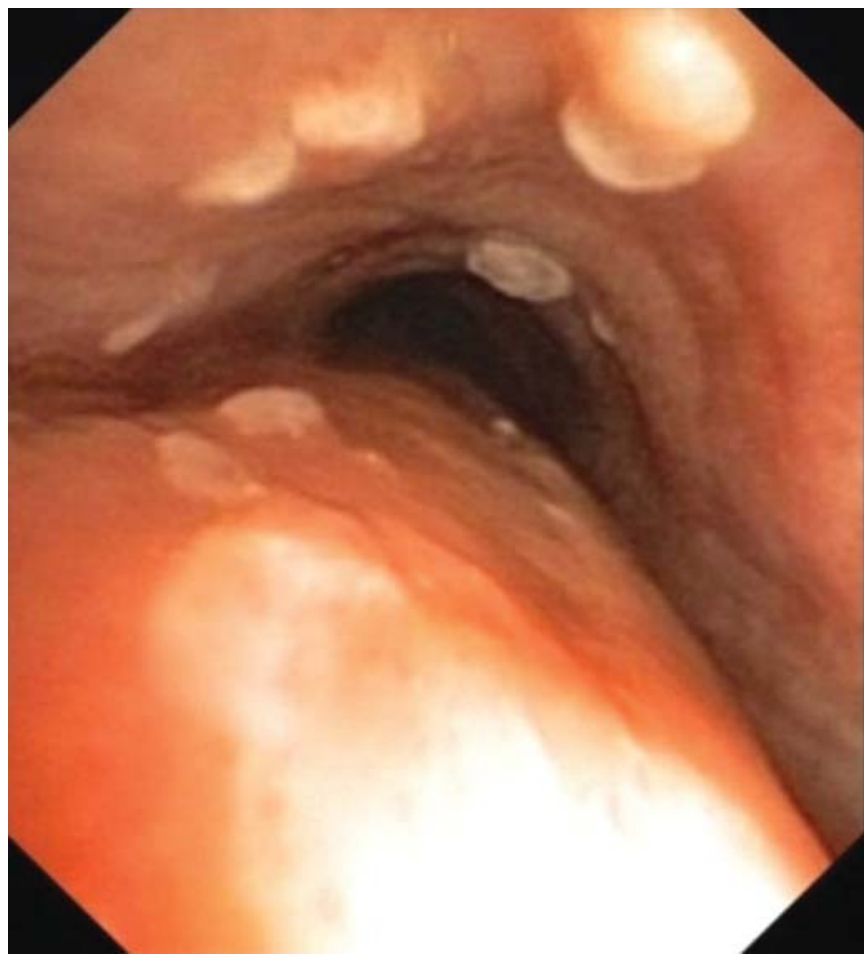

FIGURE 1. Involvement of trachea with granuloma pyogenicum after treatment with erlotinib: severe chronic bronchitis with significant breath-dependent tracheobronchial collapse. Multiple white granular mucosal lesions of the distal trachea are seen, which were biopsied. A right pneumonectomy stump without a lesion is also visible.

surfaces (60\%) [3]. In the skin, a phenomenon of satellitosis (as in our case) is described in the literature, mostly in the trunk, particularly in the scapular area [3]. Interestingly, EGFR

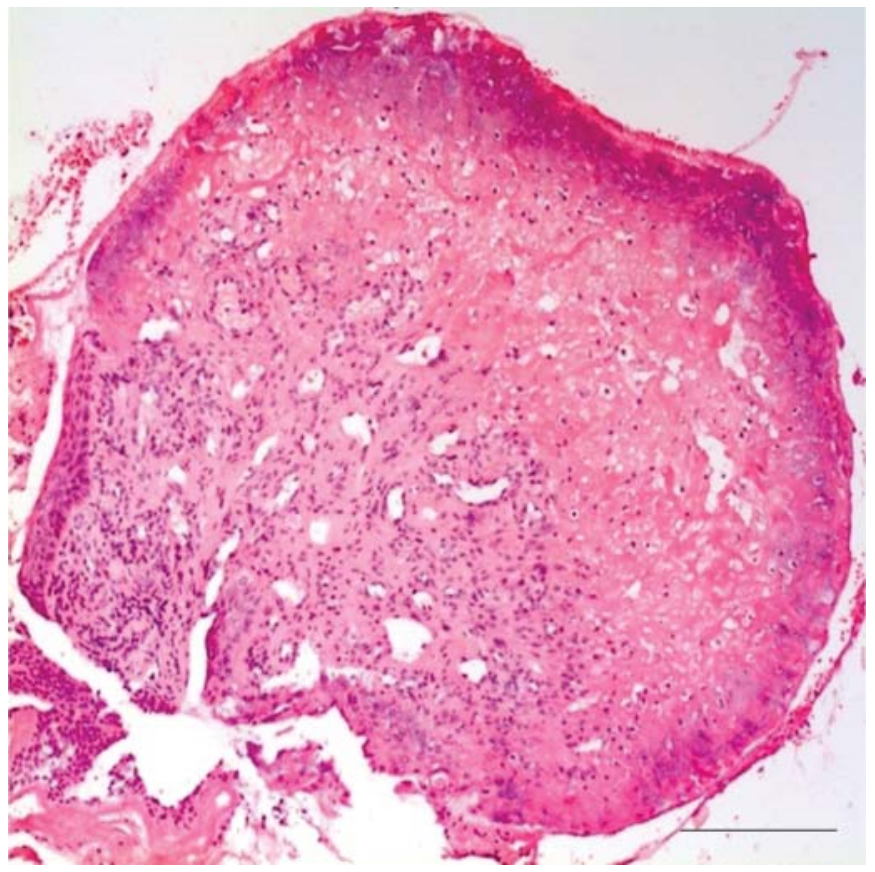

FIGURE 2. Tracheal granuloma pyogenicum. Haematoxylin and eosin staining. Scale bar $=250 \mu \mathrm{m}$.



FIGURE 3. Complete remission of granulomatous lesions in the follow-up bronchoscopy after 6 weeks.

tyrosine kinase domain mutations have also been described in normal respiratory epithelium [4]. Based on the pathological finding and the timely correlation of the described lesions in the trachea, we suspect them to be a side-effect of the erlotinib treatment. We have not previously seen a similar lesion in bronchoscopic examinations nor have we found similar cases in the literature describing endotracheal pyogenic granuloma in association with EGFR TKI treatment.

EGFR inhibitors have been reported to cause nail changes (paronychia) in up to $10-15 \%$ of treated patients, which seem to arise delayed, after weeks of treatment [5]. In several severe cases, inflammation of the nail fold was described with development of a pyogenic granuloma. Our patient had not developed any relevant symptoms or nail changes $[6,7,8,9]$.

Granulomatous changes of the trachea have been described after irritation of the airways (e.g. by a tracheostomy tube). In the presented case, the granulomata occurred $>1 \mathrm{yr}$ after the final operation and occurred diffusely along the major airways, making mechanical irritation unlikely. Multiple bronchoscopies may represent a certain irritation, yet we have never observed such a reaction before.

Although we have no proof that the tracheal pyogenic granulomas were a side-effect of erlotinib treatment, we made this diagnosis upon exclusion of other causes. In addition, the prompt disappearance of the lesions and the symptoms after stopping erlotinib further supports our hypothesis. We hope that this article encourages readers to report similar findings and consider EGFR TKIs as possible causes of tracheal granuloma pyogenicum. 
P.M. Putora*, G. Benz" ${ }^{\#}$ R. Rodriguez ${ }^{\mp}$, M. Brutsche ${ }^{\#}$ and M. Früh ${ }^{+}$

*Dept of Radiation Oncology, "Dept of Pneumology, "Institute of Pathology, and ${ }^{+}$Dept of Oncology and Hematology, Cantonal Hospital St Gallen, St Gallen, Switzerland.

Correspondence: P.M. Putora, Klinik für Radio-Onkologie, Kantonsspital St Gallen, 9007 St Gallen, Switzerland. E-mail: paul.putora@kssg.ch

Statement of Interest: None declared.

\section{REFERENCES}

1 Segaert S, Van Cutsem E. Clinical signs, pathophysiology and management of skin toxicity during therapy with epidermal growth factor receptor inhibitors. Ann Oncol 2005; 16: 1425-1433.

2 Tang X, Shigematsu H, Bekele BN, et al. EGFR tyrosine kinase domain mutations are detected in histologically normal respiratory epithelium in lung cancer patients. Cancer Res 2005; 65: 7568-7572.
3 Bolognia JL, Jorizzo JL, Rapini RP, eds. Dermatology. St Louis, Mosby, 2007.

4 Weiss SW, Goldblum JR, Enzinger FM, eds. Enzinger and Weiss's Soft Tissue Tumours. 5 Edn. St Louis, Mosby, 2008.

5 Lee MW, Seo CW, Kim SW, et al. Cutaneous side effects in nonsmall cell lung cancer patients treated with Iressa (ZD1839), an inhibitor of epidermal growth factor. Acta Derm Venereol 2004; 84: 23-26.

6 Boucher KW, Davidson K, Mirkhur B, et al. Paronychia induced by cetuximab, an antiepidermal growth factor receptor antibody. J Am Acad Dermatol 2002; 45: 632-633.

7 Dainichi T, Tanaka M, Tsuruta N, et al. Development of multiple paronychia and periungual granulation in patients treated with gefitinib, an inhibitor of epidermal growth factor receptor. Dermatology 2003; 207: 324-325.

8 Nakano J, Nakamura M. Paronychia induced by gefitinib, an epidermal growth factor receptor tyrosine kinase inhibitor. J Dermatol 2003; 30: 261-262.

9 Chang GC, Yang T-Y, Chen K-C, et al. Paronychia and skin hyperpigmentation induced by gefitinib in advanced non-small-cell lung cancer. J Clin Oncol 2004; 22: 4646-4648.

DOI: $10.1183 / 09031936.00008411$

\section{Atypical pulmonary carcinoid tumour in a 28-year-old nonsmoker with Prader-Willi syndrome}

\section{To the Editors:}

In recent years, the life expectancy of patients with Prader-Willi syndrome (PWS) has increased, unveiling several cases of earlyonset cancer and raising the question whether the condition predisposes to tumourigenesis. We encountered a unique occurrence of pulmonary atypical carcinoid in a young neversmoker with PWS.

A 28-yr-old, morbidly obese male with PWS was admitted as a candidate for gastric bypass. He was a lifetime nonsmoker, and his height, weight and body mass index were $1.78 \mathrm{~m}, 175 \mathrm{~kg}$, and $55.2 \mathrm{~kg} \cdot \mathrm{m}^{-2}$, respectively. Polyphagia, obesity and a failure to achieve psychosocial milestones became apparent at the age of 5 yrs, when PWS was diagnosed based on clinical findings and a de novo deletion of the proximal region of chromosome 15 (del(15)(q11-q13)). Type II diabetes mellitus was diagnosed at the age of $17 \mathrm{yrs}$, and dietary control along with administration of sulfonylurea was initiated. The remainder of the anamnesis included low levels of testosterone, which were treated with monthly intramuscular depot injections of testosterone enanthate $(250 \mathrm{mg})$.

On admission, the patient reported mild shortness of breath, nonproductive cough and malaise of indeterminate origin. A physical exam revealed markedly increased subcutaneous fat, picking scars, hypoplastic external genitalia and a polyphonic wheeze over the right hemithorax. Pulse was regular and of normal rate, diastolic blood pressure was elevated $(98 \mathrm{mmHg})$ and haemoglobin oxygen saturation was $96 \%$ in room air. Electrocardiography was normal. Laboratory data, including blood cell counts, routine serum biochemistry and coagulation times, were within normal limits, except for fasting plasma glucose $\left(178 \mathrm{mg} \cdot \mathrm{dL}^{-1}\right)$ and lipids (total cholesterol $233 \mathrm{mg} \cdot \mathrm{dL}^{-1}$; high-density lipoprotein cholesterol $32 \mathrm{mg} \cdot \mathrm{dL}^{-1}$; triglycerides $\left.322 \mathrm{mg} \cdot \mathrm{dL}^{-1}\right)$.

A pre-operative chest radiograph, performed routinely but also prompted by the patient's wheeze, showed a round, regularshaped opacity of soft-tissue quality measuring $7 \times 5 \mathrm{~cm}$ superimposed on the right pulmonary hilum (fig. 1a). Computed tomography confirmed a $7.5 \times 6.5 \times 5 \mathrm{~cm}$ well-demarcated mass of soft-tissue density partially attached to the posteromedial structures of the right hemithorax in very close proximity to the posterior mediastinum (fig. 1b). Fibreoptic bronchoscopy revealed a pale tumour with well-defined margins measuring $\sim 1 \mathrm{~cm}$ in diameter and obliterating the bronchus intermedius by $90 \%$ (fig. 1c). The endobronchial portion of the tumour rested on a relatively limited base and was bronchoscopically removed.

Histopathological examination of endobronchial biopsies (fig. 1d-i) showed an atypical carcinoid with focal areas of necrosis (fig. 1d), two to 10 mitoses per $2 \mathrm{~mm}^{2}$ detected by Ki67 immunostaining (fig. 1e), high cytokeratin 8 expression indicating epithelial origin (fig. 1f), and high CD56, chromogranin and synaptophysin expression indicating neuroendocrine differentiation (fig. 1g-i) [1]. There was no evidence of neoplasms of the pituitary, pancreas and parathyroid, as determined by brain magnetic resonance imaging and octreotide scanning. The tumour was pre-operatively staged as T3N0M0 (stage IIB) according to the 2009 TNM7 system, as the primary tumour measured $>7 \mathrm{~cm}$, but no locoregional or distant metastases were found [2]. Surgical resection was decided upon, and middle and lower bilobectomy was 